\section{Lichttherapie bei Pruritus: UV-B nicht mit UV-A kombinieren!}

\author{
Ein Schweizer Ärzteteam hat untersucht, ob sich aus der Kombination \\ kurzwelliger UV-B- mit längerwelliger UV-A-Strahlung ein klinisch relevanter \\ Effekt auf Juckreiz ergibt.
}

D ie Rationale hinter einer Phototherapie bei Patienten mit chronischentzündlichen Hauterkrankungen besteht unter anderem darin, dass die UV-Strahlung Zellen des Immunsystems daran hindern soll, übermäßig Histamin auszuschütten. Dadurch soll der oft sehr quälende Juckreiz gelindert werden. In-vitro-Studien legen nahe, dass sowohl UV-B- als auch UV-AStrahlen die Histaminfreisetzung aus Mastzellen inhibieren; UV-A soll darüber hinaus die Ausschüttung des Gewebshormons auch aus Basophilen eindämmen. Genau ist der Wirkmechanis- mus für beide Strahlungsarten jedoch nicht geklärt.

In einer randomisierten Studie absolvierten insgesamt 45 Patienten eine 16-wöchige Phototherapie, wobei die Sitzungen dreimal wöchentlich stattfanden. Bei 24 Teilnehmern bestand die Therapie lediglich aus UV-B-Strahlen mit einer Wellenlänge von $311 \mathrm{~nm}$; 21 Patienten bekamen UV-B- kombiniert mit UVA-Licht verabreicht; die Wellenlängen lagen hier zwischen 320 und $400 \mathrm{~nm}$.

Die Teilnehmer litten überwiegend unter atopischer Dermatitis oder Psoriasis (jeweils 37\%). Als Einschlusskriterium

\title{
Kaposi-Sarkom des harten Gaumens
}

Ein 33-jähriger Mann litt seit Monaten unter allgemeiner Müdigkeit und Leistungsschwäche. Außerdem störten ihn ausgedehnte Veränderungen an der Haut und in der Mundhöhle. Bei der Untersuchung erkannte man multiple, derbe, nicht druckdolente, livide Plaques am harten Gaumen. Ähnliche Veränderungen fanden sich auch im Gesicht, an der Thoraxwand und an den Beinen. Im Labor fielen eine ausgeprägte Leukopenie mit 2.900 Zellen/ $\mu$ l und eine CD4-Zellzahl von 76/ $\mu$ l auf. Unter der Diagnose einer HIV-Infektion entnahm man Biopsien aus der Haut und den Läsionen in der Mundhöhle, die eine spindelzellartige Neoplasie mit positiver Expression von CD34 und humanem Herpesvirus 8 (HHV-8) bei der immunhistochemischen Testung zeigten. Diese Befunde sprachen für ein Kaposi-Sarkom. Man begann eine antiretrovirale Therapie (ART) und eine Chemotherapie.

Das Kaposi-Sarkom ist eine Neoplasie, die praktisch nur bei Patienten mit ausgeprägtem zellulärem Immundefekt vorkommt. Ausgelöst wird es durch HHV-8. Es kann

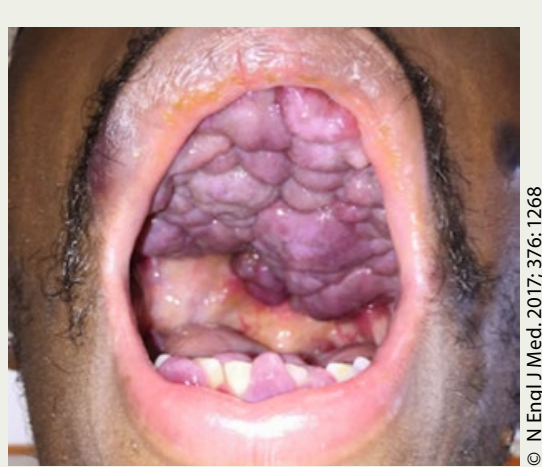

Derbe, livide Plaques am harten Gaumen. isoliert auf der Haut auftreten, aber auch innere Organe befallen, vor allem die Mundhöhle, den Gastrointestinal- und den Respirationstrakt.

Ein derart ausgedehnter Befund wie im vorliegenden Fall ist mit einer schlechten Prognose assoziiert. Dennoch ging die Tumorgröße bei dem Patienten nach mehreren Monaten Therapie zurück. Unter fortlaufender ART stieg die CD4Zellzahl an, und der Patient fühlte sich besser. Prof. Hermann S. FüeßI

Kennedy-Le Jeune E et al. Kaposi's sarcoma of the oral cavity. N Engl J Med. 2017; 376: 1268 galt ein Juckreizschweregrad von mindestens 5 auf der 10 Punkte umfassenden visuellen Analogskala (VAS). Zur Beurteilung des Therapieerfolgs wurde zusätzlich der 5-D-Itch-Score herangezogen. $\mathrm{Zu}$ Beginn lagen die VAS-Werte in der UVB-Gruppe bei durchschnittlich 7,2, in der Gruppe mit der UV-B-/UV-A-Kombination bei 7,0. Die Werte im 5-D-Itch-Score betrugen 20,9 und 19,8. Beides unterschied sich nicht nennenswert.

Nach 16 Wochen Bestrahlung hatte sich der Juckreiz in beiden Gruppen verbessert, in der UV-B-Gruppe auf einen Wert von 2,0 Punkten im VAS-Score, in der Kombinationsgruppe auf 2,5 Punkte; ein signifikanter Unterschied, ob mit oder ohne UV-A bestrahlt worden war, bestand damit nicht. Beim 5-D-ItchScore - ging der Wert auf 10,5 Punkte in der UV-B- und 13,3 Punkte in der UV-B/ UV-A-Gruppe zurück, was ebenfalls nicht signifikant war.

Neben dem Juckreiz hatte sich auch die Krankheitsaktivität in beiden Gruppen deutlich gebessert, aber ebenfalls ohne signifikanten Unterschied: Von einem Ausgangswert von 12,9 in der UV-B- und 11,4 in der Kombinationsgruppe sank die Krankheitsaktivität in den 16 Behandlungswochen auf letztlich 2,2 und 1,7. Vergleichbare Effekte wurden auch beim Parameter Lebensqualität erzielt: Im DLQI (Dermatology Quality of Life Index) gingen die Werte von 11,2 und 15,5 auf 5,8 und 6,5 zurück.

In der Studie war es in der Kombinationsgruppe in zwei Fälle zur Exazerbation der Hauterkrankung gekommen. Dagegen waren in der rein mit UV-B bestrahlten Gruppe Nebenwirkungen ausgeblieben.

Fazit: : Bei Patienten mit Psoriasis oder atopischem Ekzem bringt es für den Juckreiz offenbar nichts, eine Phototherapie mit UV-B-Strahlen durch einen UV-A-Anteil zu ergänzen. Angesichts möglicher kumulativer Nebenwirkungen empfehlen die Autoren, die Indikation für den UV-A-Zusatz restriktiv zu stellen.

Dr. Elke Oberhofer

Maul JT et al. Impact of UVA on pruritus during UVA/B phototherapy of inflammatory skin diseases: a randomized double-blind study. J Eur Acad Dermatol Venereol 2017; 31: 1208-13 